\title{
Triple Helix Formation of Procollagen Type I Can Occur at the Rough Endoplasmic Reticulum Membrane*
}

(Received for publication, February 7, 1996, and in revised form, April 15, 1996)

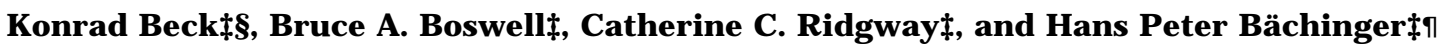 \\ From the $¥$ Shriners Hospital for Children, Research Unit, Portland, Oregon 97201 and the 1 Department of Biochemistry \\ and Molecular Biology, Oregon Health Sciences University, Portland, Oregon 97201
}

\begin{abstract}
One key problem in understanding the biosynthesis of collagens remains the assembly of the three $\alpha$-chains. How and when are the different gene products selected, aligned, and folded into a triple helix? As the spatial arrangement during biosynthesis might be important, we concentrated on whether the rough endoplasmic reticular membrane is involved in this process. Microsomes were prepared from biosynthetically labeled chick tendon fibroblasts. Vesicles were spread as a monomolecular film which was then transferred over several compartments of a filmbalance containing fresh subphase. Fluorograms of the surface film showed that the monolayer contains procollagen chains. When the monolayer was transferred onto a chymotrypsin/trypsin-containing subphase, the gel bands of the pro $\alpha$ chains were shifted into the position of mature $\alpha$-chains, indicating that only the propeptides were digested and the collagenous regions were protected due to triple helix formation. Our results suggest that newly synthesized pro $\alpha$-chains can associate as trimers and fold into a triple helical conformation while they are still associated with the membranes of the rough endoplasmic reticulum. These processes also occur when interchain disulfide linkage is inhibited, indicating that chain selection and registration is not dependent on formation of covalent bonds among the carboxyl propeptides.
\end{abstract}

Collagens are the major structural components of the extracellular matrix (for a recent review, see R ef. 1, and references therein). The polypeptide chains containing a varying number of repeating Gly-Xaa-Yaa repeating units with proline commonly found in the $X a a$ and hydroxyproline frequently located in the Yaa position assemble into triple helices due to the glycine residues in each third position which fit into the center of the helix. The most common of the more than 19 different types of collagens is collagen I which is normally composed of two $\alpha 1$ - and one $\alpha 2$-chains (2). It is found in most connective tissues, except some cartilages and basement membranes. Trimeric collagen type I molecules assemble into fibrils by headto-tail arrangement and laterally staggered alignment.

The current view of the intracellular steps of collagen biosynthesis (for a review see, eg. Ref. 3) suggests that translation

* This work has been supported by grants from the Shriners Hospital for Children. The electron microscopy facility received additional support from the Fred Meyer Charitable Trust Foundation and the R. Blaine Bramble Medical Research Foundation. The costs of publication of this article were defrayed in part by the payment of page charges. This article must therefore be hereby marked "advertisement" in accordance with 18 U.S.C. Section 1734 solely to indicate this fact.

$\S$ To whom correspondence should be addressed: Shriners Hospital for Children, Research Unit, 3101 S. W. Sam J ackson Park Rd., Portland, OR 97201. Tel.: 503-221-3448; Fax: 503-221-3451; E-mail: KGB@SHCC.ORG. of the pre-procollagen mRNA starts on nonmembrane bound ribosomes. After the signal peptide forms an intermediate complex with a signal recognition particle, it associates with the $E R{ }^{1}$ Once in the ER, the nascent chains undergo extensive cotranslational modifications (4), especially hydroxylation of specific peptidyl lysine and proline residues, which is followed by glycosylation of certain hydroxylysyl residues $(5,6)$. Assembly of the pro $\alpha$-chains into procollagen trimers is initiated by the folding of the C-propeptides of the individual chains and formation of intrachain disulfide bonds, association of the Cpropeptides followed by interchain disulfide bond formation (7, 8), nucleation of the $\mathrm{Col} 1$ domain and propagation of the triple helix in a zipper-like action from the $\mathrm{COOH}$ to $\mathrm{NH}_{2}$ terminus (9-11). The correct sets of three carboxyl propeptides of procollagen I and procollagen III are also formed when triple helix formation is prevented by inhibition of proline hydroxylation (12-14). The carboxyl propeptides are able to fold separately, followed by recognition and assembly to the trimeric complex. The telopeptide region between the carboxyl propeptides and the start of the collagen helix region are essential for trimer formation in vitro (15). Helix formation is interrupted by the random occurrence of cis peptide bonds which are converted into the trans configuration by peptidyl-prolyl cis-trans isomerase in vitro $(16,17)$ and probably also in vivo $(18,19)$. The amino-terminal propeptides then associate and form the short stretch of triple helix within these peptides.

An open question in the biosynthetic pathway remains of how and when the different gene products are selected, aligned, and subsequently folded into a triple helix. As the spatial arrangement during biosynthesis is perhaps of relevance, we tried to determine whether the rough $\mathrm{ER}$ membrane is involved in chain recognition and association of the three pro $\alpha$-chains. As the maximum rate of a reaction depends on the encounter probabilities of the components, it would be advantageous if at least the initial steps of collagen folding would occur while the single chains are still associated with the internal surface of the lumen of the $E R$. A reduction of dimensionality has been proposed to be of general advantage for multimolecular diffusion-controlled processes, especially at low concentrations (20). Based on their analysis of the folding of the carboxyl-terminal propeptide, such a mechanism has been pointed out by Doege and Fessler (15) and is supported by the finding that the components of a triple helical molecule are made in close proximity (21).

We have approached this problem by spreading rough ERderived vesicles containing biosynthetically label ed procol lagen chains as monolayers at the air/water interface. After transferring the monolayer onto fresh subphase we found that procollagen chains were co-transported with the membrane. Testing

\footnotetext{
${ }^{1}$ The abbreviations used are: ER, endoplasmic reticulum; NEM, Nethylmaleimide; PMSF, phenylmethanesulfonyl fluoride; POPC, 1-palmitoyl-2-ol eoyl-L- $\alpha$-sn-3-glycero-phosphatidylchol ine.
} 
for the structural arrangement of the polypeptide chains revealed that in this state procollagen is already folded into their triple helical structure. Therefore we propose that trimerization occurs when the single pro $\alpha$-chains are still attached directly or indirectly to the ER membrane.

\section{MATERIALS AND METHODS}

Reagents-If not otherwise indicated, all chemicals were from commercial suppliers and used without further purification. Collagenase (Worthington; CLSPA grade) was further purified by size exclusion chromatography on a Superose 12 column (Pharmacia Biotech Inc., Uppsala, Sweden) equilibrated in $50 \mathrm{~mm}$ Tris- $\mathrm{HCl}, 5 \mathrm{~mm} \mathrm{CaCl}$, pH 7.6 (22). Column fractions eluting as the first peak were concentrated by ultrafiltration. On SDS gels, this material shows a single band with an apparent $M_{r}$ of $\sim 115,000$. Concentrations were determined spectroscopically assuming $\epsilon_{280}=1.67 \times 10^{5} \mathrm{M}^{-1} \mathrm{~cm}^{-1}$ (23). $\left[{ }^{14} \mathrm{C}\right] \mathrm{Pro}$, [ $\left.{ }^{35} \mathrm{~S}\right] \mathrm{Met}$, and $\left.{ }^{35} \mathrm{~S}\right]$ Cys were from DuPont NEN. Lipids were from Avanti Polar Lipids (Alabaster, AL). Purity and lipid composition of rough ER vesicles were checked by thin layer chromatography followed by staining in iodine vapor. Buffers were prepared with MilliQ water. For monolayer experiments, buffers were filtered $(0.22 \mu \mathrm{m})$ and degassed.

Preparation of Rough ER Chicken Fibroblast Vesicles-The tendon cell preparation basically followed established protocols $(24,25)$. Briefly, leg tendon cells from $\sim 70$ eggs of 17-day-old chick embryos were prepared by enzymic digestion. Digested tendons were filtered through lens tissue, pelleted, washed, and resuspended in the same medium with the addition of $10 \%$ fetal calf serum. Cells were incubated in this medium for at least 30 min prior to any further treatment. For biosynthetic labeling, cells were pelleted and washed in medium lacking cysteine, proline, and methionine and resuspended. About $1 \times 10^{9}$ cells were incubated at $7 \times 10^{6} \mathrm{cell} / \mathrm{s} / \mathrm{ml}$ at $37^{\circ} \mathrm{C}$ for $15 \mathrm{~min}$. Labeling was carried out in volumes of $10 \mathrm{ml}$ for $7.5 \mathrm{~min}$ or overnight. Label concentrations were $1 \mathrm{mCi} / \mathrm{ml}\left[{ }^{35} \mathrm{~S}\right] \mathrm{Met}$ and $\left[{ }^{35} \mathrm{~S}\right] \mathrm{Cys}$, and $100 \mu \mathrm{Ci} / \mathrm{ml}\left[{ }^{14} \mathrm{C}\right] \mathrm{Pro}$.

To prevent stable triple helix formation by inhibition of hydroxylation, in some experiments cells were incubated overnight in medium containing $3 \mathrm{~mm} \alpha, \alpha^{\prime}$-dipyridyl, and labeling was performed as described above.

Cells were washed and resuspended twice at approximately 5 times the cell pellet volume in phosphate-buffered saline without divalent cations. To inhibit disulfide linkage, NEM was added to the buffer to a final concentration of $1 \mathrm{~mm}$. Cells were resuspended in homogenization buffer ( $5 \mathrm{~mm}$ Hepes, pH 6.8, 250 mm sucrose, 1 mm PMSF, 1 mM NEM, $1 \mathrm{~mm}$ leupeptin) at 5 times the cell volume (26). This suspension was frozen in liquid nitrogen and stored at $-130^{\circ} \mathrm{C}$

For vesicle preparation, the cell suspension was thawed on ice and lysed in a 15- $\mathrm{ml} \mathrm{N}_{2}$ cavitation bomb (Kontes Co., Millville, $\mathrm{NJ}$ ) at 3.5 bar for $15 \mathrm{~min}$ (27). The suspension was extruded into a homogenizer vessel, adjusted to $1 \mathrm{~mm} \mathrm{Mg}^{2+}$, and then motor dounced at a very slow speed with five strokes of a Teflon pestle. The cell debris was pelleted at $1000 \times \mathrm{g}$. The supernatant was transferred to a new tube, and the pellet was resuspended in $2 \mathrm{ml}$ of the buffer that was used to wash the bomb and that had been adjusted to $1 \mathrm{~mm} \mathrm{Mg}^{2+}$. The pellet was briefly homogenized again and repelleted. To pellet mitochondria, the supernatants were pooled and centrifuged at $10,000 \times \mathrm{g}$ for $10 \mathrm{~min}$. The supernatant was transferred to a SW40Ti UItra-Clear tube (Beckman). The pellet was resuspended in $2 \mathrm{ml}$ of buffer as before but without homogenizing and repelleted. The combined supernatants were underlayed with $2 \mathrm{ml}$ each of 0.44 and $1.3 \mathrm{M}$ sucrose in the same buffer. Rough ER microsomes were sedimented through both steps at $105,000 \times \mathrm{g}$ for $2 \mathrm{~h}$.

For stripping off the ribosomes, the microsomes were resuspended in $4 \mathrm{ml}$ of $5 \mathrm{~mm}$ Hepes, $\mathrm{pH} 6.5,50 \mathrm{~mm} \mathrm{KCl}$ and pelleted at $25,500 \times \mathrm{g}$ for $15 \mathrm{~min}$. The pellet was resuspended in $1 \mathrm{ml}$ of the same buffer, the absorbance at $260 \mathrm{~nm}$ was measured, and $200 \mathrm{~mm}$ EDTA was added to a final amount of $0.3 \mu \mathrm{mol}$ per absorbance unit per $\mathrm{cm}$ (28). Effective monolayer generation was usually achieved if the absorbance at this step was between 1.5 to $2.0 \mathrm{OD}$. The stripped vesicles were repelleted, stored on ice, and used immediately for monolayer experiments.

Monolayer Experiments-Monolayer experiments were performed on a multicompartment trough (model RMC2-T, Mayer Feintechnik, Göttingen, FRG) built according to the design of Fromherz (29). The trough design differs from conventional Langmuir film balances in that it uses a circular geometry. The trough area is enclosed within two circles with inner and outer diameters of 130 and $250 \mathrm{~mm}$, respectively, and divided into eight equal parts each covering a $45^{\circ}$ sector by knife edged Teflon spacers. The monolayer is further enclosed between two barriers movable by a feedback-controlled motor device located in the center of the trough. One of the barriers holds a high frequency displacement transducer (Collins, Long Beach, CA, model SS-101) coupled to a spring which measures the surface tension by the Wilhelmy method. The surface pressure $\Pi$ is defined as the difference of the surface tension of the pure subphase and the monolayer covered subphase relating the readily measurable change in surface pressure with the intermolecular forces of the monomolecular film (for details, see eg. Ref. 30). Most of the experiments reported in this study were carried out in the constant surface pressure mode, i.e any surface pressure increase due to vesicle spreading was compensated by a feedback controlled surface area increase which was monitored as an area change. To avoid leakage of the monolayer at the edges of the Teflon trough, the barriers were milled from Kel-F. The surface pressure was measured with a Wilhelmy plate made of roughened platinum/iridium (Krüss, Hamburg, FRG) with a circumference of $4 \mathrm{~cm}$. The instrument was enclosed in a cabinet mounted on a massive granite block which sat on rubber feet to reduce vibrations. Surface pressure and area data were collected with a sampling rate of $2 \mathrm{~s}$ by a computer. After each experiment the trough, barriers, glass rod, and Wilhelmy plate were rigorously cleaned with detergent (RBS 35; Pierce), ethanol, and water. At the beginning of each experiment, the absence of surface active components was checked by compressing the full surface area of the subphase buffer to a minimum area of $15 \mathrm{~cm}^{2}$, which results in a surface pressure increase of less than $1 \mathrm{mN} / \mathrm{m}$. All experiments were carried out at room temperature $\left(20-23^{\circ} \mathrm{C}\right)$.

Lipids were dissolved in hexane $(\mathrm{c}=1 \mathrm{mg} / \mathrm{ml})$ and $5 \mu \mathrm{l}$ were spread to an area of $100 \mathrm{~cm}^{2}$. After allowing the solvent to evaporate, the monolayer was slowly compressed $\left(\sim 20 \mathrm{~cm}^{2} / \mathrm{min}\right)$ to the desired surface pressure which was held constant for the rest of the experiment. The rough ER vesicle pellet (see above) was resuspended in 10-15 $\mu \mathrm{l}$ of subphase buffer by gentle vortexing for $\sim 30 \mathrm{~s}$. The vesicle spreading technique is reminiscent of the procedure described by Trurnit (31) (see also Verger and Pattus (32)). The vesicle suspension was applied to a wet glass rod ( $3 \mathrm{~mm}$ in diameter) in $2-\mu \mathrm{l}$ droplets. The glass rod was washed with 20-50 $\mu \mathrm{l}$ of subphase buffer and submerged into the subphase.

The monolayer was incubated on the initial subphase for 90-120 min after which it was transferred onto the same buffer (control) or a buffer containing proteases (see below) where it was incubated for $120 \mathrm{~min}$. Transfer was performed by switching off the constant surface pressure feedback control and moving the monolayer by simultaneous displacement of the two barriers in a clockwise or anticlockwise direction. Finally, the monolayer was transferred onto fresh buffer without enzymes and recovered by aspiration into tubes resulting in a volume of $2-3 \mathrm{ml}$. Completeness of aspiration of the monolayer was ascertained by the decay of the surface pressure to zero at minimal area. In the case of protease experiments, these tubes contained 1-ml solutions of either 1 mM EDTA (to inhibit collagenase), $1 \mathrm{~mm}$ PMSF (to inhibit trypsin and chymotrypsin), or $1 \mathrm{~m}$ ammonium bicarbonate (to inhibit pepsin). The complete initial spreading subphase as well as the enzyme-containing subphases were collected directly into lyophilizing flasks. All solutions were frozen to $-130^{\circ} \mathrm{C}$ and repeatedly lyophilized. The resulting material was analyzed by fluorography.

Subphase buffers were $0.2 \mathrm{M}$ ammonium acetate, $0.1 \mathrm{~mm} \mathrm{CaCl}, \mathrm{pH}$ 6.8, except for experiments involving pepsin when $0.1 \mathrm{~m}$ acetic acid, $\mathrm{pH}$ 2.7, was used. Enzymes were dissolved in the subphase buffer directly before use to concentrations of $5 \mu \mathrm{g} / \mathrm{ml}$ collagenase, $100 \mu \mathrm{g} / \mathrm{ml}$ chymotrypsin $/ 10 \mu \mathrm{g} / \mathrm{ml}$ trypsin, or $5 \mu \mathrm{g} / \mathrm{ml}$ pepsin.

SDS-Polyacrylamide Gel Electrophoresis and Fluorography-Gel electrophoresis was performed using 5-20\% polyacrylamide gradient gels (33). Samples were applied either unreduced or reduced with 20 $\mathrm{mm}$ dithiothreitol. For fluorography, gels were fixed and soaked in Amplify (Amersham Corp.) for 20 min. Dryed gels were incubated on preflashed Biomax MR film (Kodak) for $1-50$ days at $-80^{\circ} \mathrm{C}$.

Electron Microscopy-Ultrathin sections of rough ER vesicles before and after stripping off the ribosomes were analyzed by electron microscopy. Vesicles were washed and resuspended in $0.1 \mathrm{~m}$ sodium cacodylate buffer, pH 7.4, and prepared for thin sectioning as described previously (34).

Vesicle-derived monolayers were analyzed by negative stain electron microscopy. 600 mesh carbon-coated grids were used without glowdischarge and thus were rather hydrophobic. They were carefully placed on different monolayer areas for $10-60 \mathrm{~s}$ and picked up without immersing them into the subphase. Grids were stained for 1 min with $2 \%(w / v)$ phosphotungstic acid. 


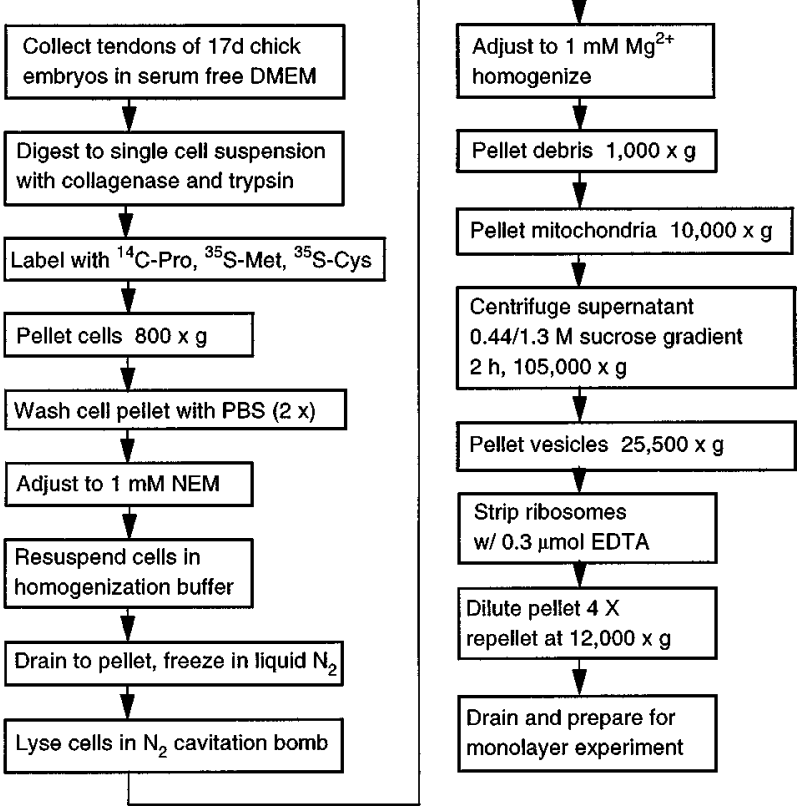

FIG. 1. Flow chart of the preparation of rough ER vesicles from embryonic chick tendon fibroblasts. See text for details.

RESULTS

Preparation of Rough ER Vesicles-To obtain sufficient vesicle material containing procollagen type I chains for use in monolayer experiments, we established a preparation protocol by combining various techniques described (see "Materials and Methods") (Fig. 1). Fibroblasts are grown from tendons of 17day chick embryos. For most experiments cells are label ed with radioactive proline, cysteine, and methionine for $7.5 \mathrm{~min}$. Based on pulse-chase experiments, this is about the time required for the synthesis of a complete pro $\alpha$-chain $(35,36)$. To diminish the formation of interchain disulfide linkage during the following preparation steps and during monolayer experiments, cells were briefly treated with 1 mM NEM. After washing, the cells were lysed in an $\mathrm{N}_{2}$ cavitation bomb. This step was essential as former homogenization attempts employing a Potter-Elvehjen homogenizer used in the preparation of rough $E R$ vesicles from other tissues resulted in low yields. This might be due to the extensive cytoskel eton network present in this tissue. After removing cell debris and mitochondria, rough $E R$ vesicles were collected by sucrose density centrifugation and stripped off the ribosomes by EDTA treatment.

Vesicles were analyzed for their lipid composition by thin layer chromatography. Although this method does not al low for precise quantification, the majority of lipids migrate in the position of phosphatidylcholines both before and after stripping off the ribosomes as judged from the spot intensity after incubation in iodine vapor. Furthermore, significant amounts are found in positions corresponding to (in the order of spot intensity) phosphatidylethanolamine, phosphatidylinositol, and sphingomyelin. This composition is in agreement with that reported for rough ER membranes prepared from different sources (37).

Electron microscopy of vesicles after thin sectioning (Fig. 2) revealed that the EDTA treatment effectively removed the ribosomes. Vesicles appeared unilamellar with a mean diameter of $350 \mathrm{~nm}$

Monolayer Spreading of Rough ER Vesicles-All monolayer experiments were carried out using volatile subphase buffers which allowed an analysis of the distribution of Iabeled procollagen chains by SDS-gel electrophoresis and subsequent fluorography. $100 \mu \mathrm{M} \mathrm{CaCl}$ was added which corresponds to about

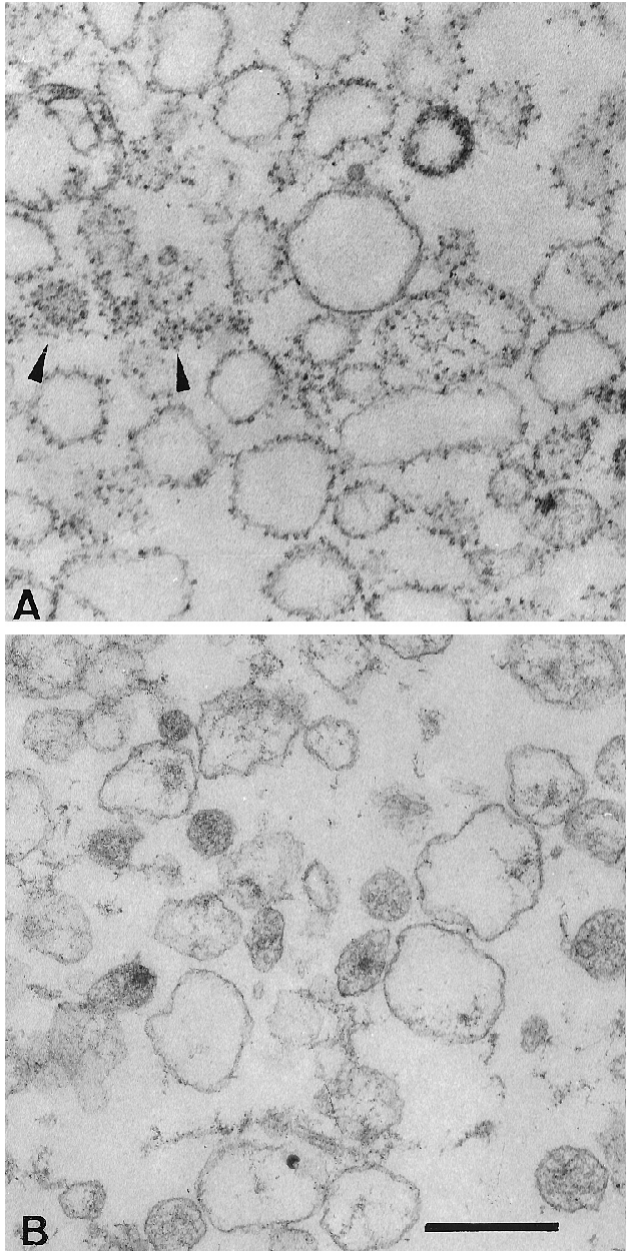

FIG. 2. Electron micrographs of rough $\mathbf{E R}$ vesicles. Vesicles were visualized by thin sectioning before (A) and after (B) stripping off the ribosomes. Arrowheads in A point to areas which represent top views of vesicles due to the finite thickness of the sections (bar, $500 \mathrm{~nm}$ ).

the concentration of free calcium in the ER lumen at rest (38). For many of the proteins investigated by monolayer techniques it was found that they undergo surface denaturation when spread at low surface pressures $(32,39)$. F or several membrane proteins denaturation can be effectively prevented when the fil $\mathrm{m}$ is generated and held at surface pressures greater than 15 $\mathrm{mN} / \mathrm{m}$ (39). Therefore we spread our vesicles against the pressure generated by a preformed lipid monolayer. POPC was chosen as a lipid as it was shown that phosphatidylcholine does not specifically interact with procollagen (40). POPC monolayers are well characterized and are in a liquid expanded state with no phase transition in the $1-45 \mathrm{mN} / \mathrm{m}$ range at room temperature (41).

In order to determine the limiting surface pressure above which no further vesicle spreading can be expected, we spread vesicles at constant area conditions against POPC monolayers of different starting surface pressures $\Pi_{i}(\mathrm{Fig}$. 3A). A quasiconstant final surface pressure $\Pi_{\mathrm{f}}$ was reached after 90-120 min. In a first approximation, the surface pressure difference $\Delta \Pi=$ $\Pi_{f}-\Pi_{i}$ is nearly inversely proportional to $\Pi_{i}$. Extrapolation to $\Delta \Pi=0$ reveals a limiting pressure of $\sim 30 \mathrm{mN} / \mathrm{m}$ independent of the initial surface pressures in the 5 to $20 \mathrm{mN} / \mathrm{m}$ range. The linear relationship of $\Delta \Pi$ versus $\Pi_{i}$ indicates that no significant unfolding of polypeptide chains occurs at low $\Pi_{i}$ which would result in unproportionally higher values of $\Delta \Pi$. The processes involved in the generation of monolayers from bilayer vesicles are only partially understood $(39,42)$. Interestingly, our limit- 

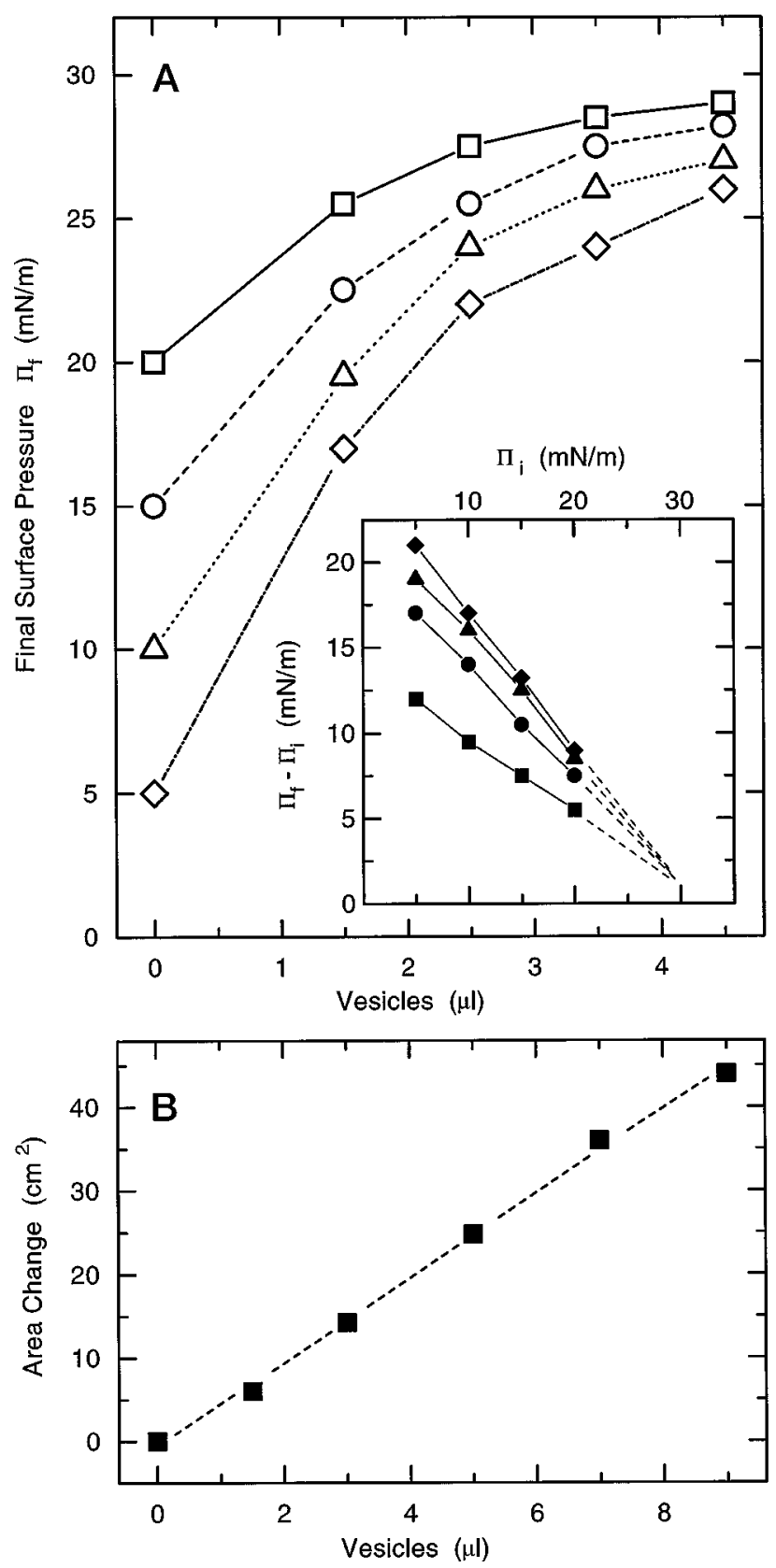

FIG. 3. Spreading of rough $\mathbf{E} \mathbf{R}$ vesicles. A, vesicle solutions of one preparation are spread successively to a preformed POPC monolayer at a constant surface area and the surface pressure increase with time is monitored. Data points represent the final surface pressure $\Pi_{\mathrm{f}}$ recorded after 60-120 min when no further significant pressure increase could be observed. The initial surface pressures $\Pi_{i}$ of the POPC monolayer are 20 ( $\square \square \square), 15(\bigcirc-\cdots--\bigcirc), 10(\triangle \cdots \cdots \triangle)$, and $5(\diamond \cdots-\cdot \diamond) \mathrm{mN} / \mathrm{m}$. The inset shows the same data points plotted as $\Pi_{i}$ versus $\left(\Pi_{f}-\Pi_{i}\right)$ where the different lines represent a total vesicle spreading volume of 1.5 $(\square), 2.5(-\bullet), 3.5(-\mathbf{\Delta})$, and $4.5(\bullet-\mu$. B, vesicles are spread successively to a preformed POPC monolayer at a constant surface pressure of $20 \mathrm{mN} / \mathrm{m}$ and the area increase with time is re corded. Data points represent the final area increase observed after 90-120 min (see also Fig. 4).

ing surface pressure is within the range of $25-35 \mathrm{mN} / \mathrm{m}$ which is frequently thought of as an equivalence surface pressure for the comparison of mono- and bilayer data (42-44).

When spread against a preformed POPC monol ayer held at a constant surface pressure of $20 \mathrm{mN} / \mathrm{m}$, the area increase is proportional to the amount of vesicles applied (Fig. 3B). This indicates that there is no noticeable interaction of the vesicle material with POPC. Spreading efficiency, however, varied

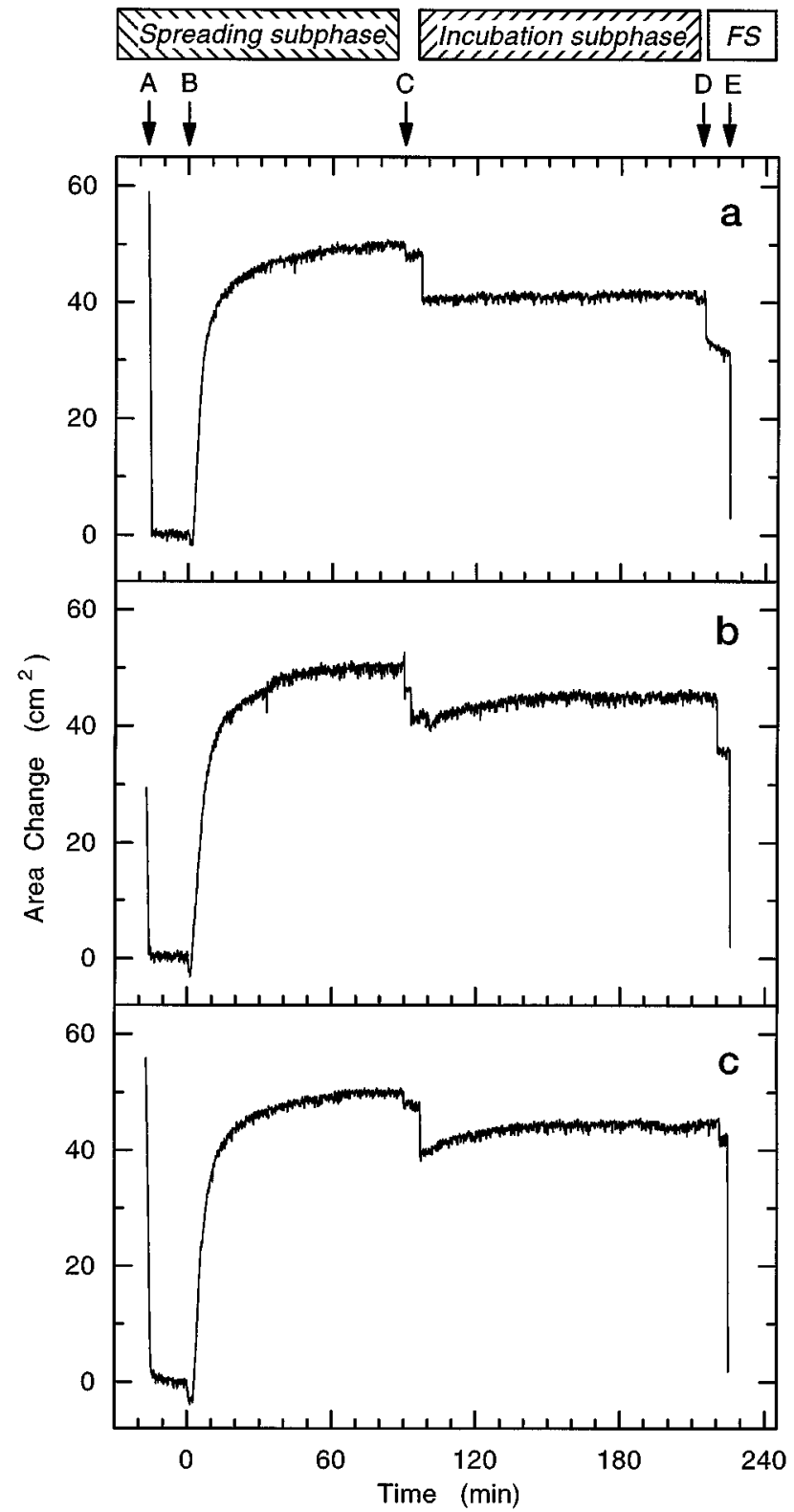

FIG. 4. Time course of monolayer experiments. $\sim 5 \mu \mathrm{g}$ of POPC are spread on an initial area of $100 \mathrm{~cm}^{2}$, and the lipid monolayer is slowly compressed to a surface pressure of $20 \mathrm{mN} / \mathrm{m}\left(\sim 30 \mathrm{~cm}^{2}\right)$ which is held constant throughout the experiment (A). The film is allowed to equilibrate for $15 \mathrm{~min}$, after which the glass rod is wetted with $20-50 \mu \mathrm{l}$ of subphase buffer, and 5-10 $\mu \mathrm{l}$ of vesicle solution are applied in 2- $\mu \mathrm{l}$ droplets within $1 \mathrm{~min}(\mathrm{~B})$. The glass rod is washed with $20-50 \mu$ l of subphase buffer and then immersed into the subphase to allow free movement of the barriers over the surface. The area change due to vesicle spreading is monitored. $90 \mathrm{~min}$ after vesicle application (C), the monolayer is transferred by $180^{\circ}$ (corresponding to $\sim 180 \mathrm{~cm}^{2}$ ) by simultaneous movement of the two barriers onto either the same subphase buffer (control, a), or buffer containing trypsin/chymotrypsin (b) or collagenase (c). As during transfer the surface pressure drops slightly (3-5 $\mathrm{mN} / \mathrm{m}$ ) indicating loss of material, the monolayer is recompressed to $20 \mathrm{mN} / \mathrm{m}$. The monolayer is incubated on the control or enzyme containing subphase for $2 \mathrm{~h}$, after which it is transferred again by $180^{\circ}$ onto a fresh subphase (D) and collected by suction (E).

crucially with the age of vesicle. When stored overnight either at $4{ }^{\circ} \mathrm{C}$ or after freezing and thawing, less than half of the area increase could be observed.

To analyze the structure of the monol ayer, we spread vesicles at a constant surface pressure of $20 \mathrm{mN} / \mathrm{m}$ and waited for $1.5 \mathrm{~h}$ up to no further substantial area increase could be observed (Fig. 4a). Then we transferred the monolayer, enclosed between 

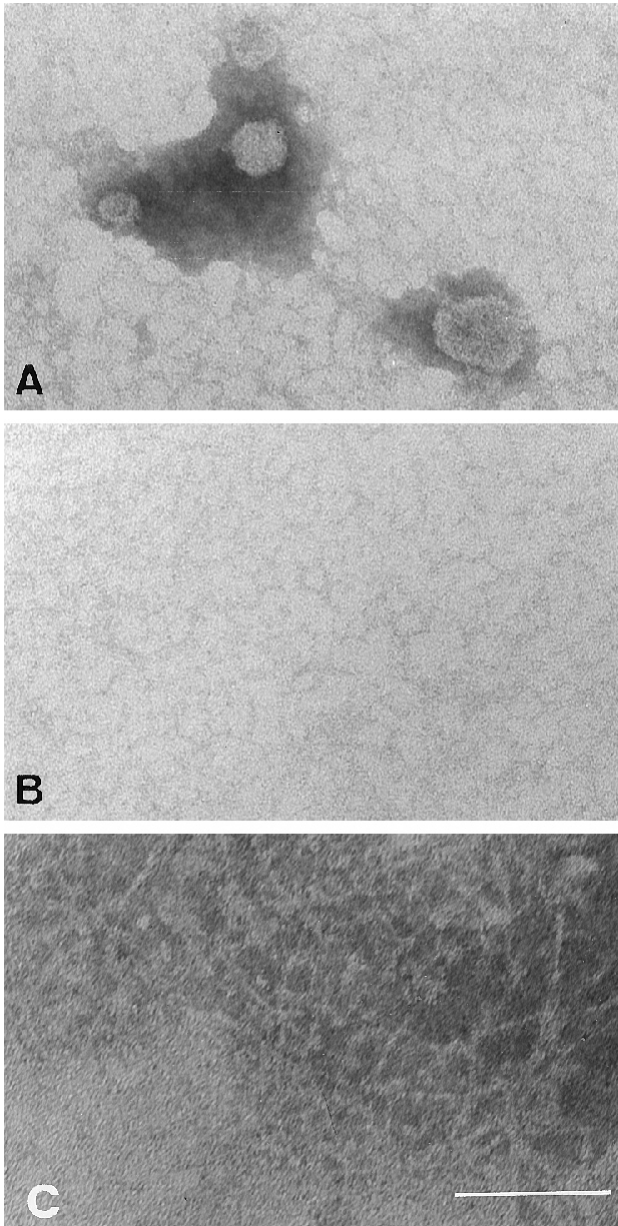

FIG. 5. Negative stain electron micrographs of monolayers derived from rough $\mathbf{E R}$ vesicles. The monolayer was picked up from the spreading subphase (A) or after transport onto fresh subphase (B). In the latter case, some areas show a fibrillar meshwork underneath the monolayer (C) (bar, $100 \mathrm{~nm})$.

two barriers, over several trough compartments onto fresh subphase, where it was incubated for $2 \mathrm{~h}$. Then the monolayer was transported again onto fresh subphase. Monolayer material from the spreading subphase taken just before transportation and after incubation on the fresh subphase was analyzed by negative stain electron microscopy. After spreading was complete, only a few vesicles could be visualized (Fig. 5A). After transfer, the monolayer appeared completely homogeneous (Fig. 5B). This indicates that any vesicles which have not integrated into the monolayer were effectively removed during transportation. In some areas, in which the monolayer appears broken, meshwork-like structures appear which may correspond to assembled procollagen molecules (Fig. 5C). Our observation that only few unspread vesicles could be found after monolayer transfer contrasts to other studies in which more sophisticated techniques for the removal of unspread vesicles were used (39). This may be due to the difference in the spreading technique and the considerably longer time allowed for equilibration.

To test whether radioactively labeled proteins adhere to the monolayer, we performed the same kind of experiment (Fig. 4a), but collected the final monolayer as well as the spreading subphase and analyzed it by fluorography (Fig. 6). The most prominent bands observed in the vesicle preparation migrate in the position of $\alpha 1$ - and $\alpha 2$-procollagen I chains under both reducing and nonreducing conditions indicating that the interchain disulfide bridges within the carboxyl propeptide have not
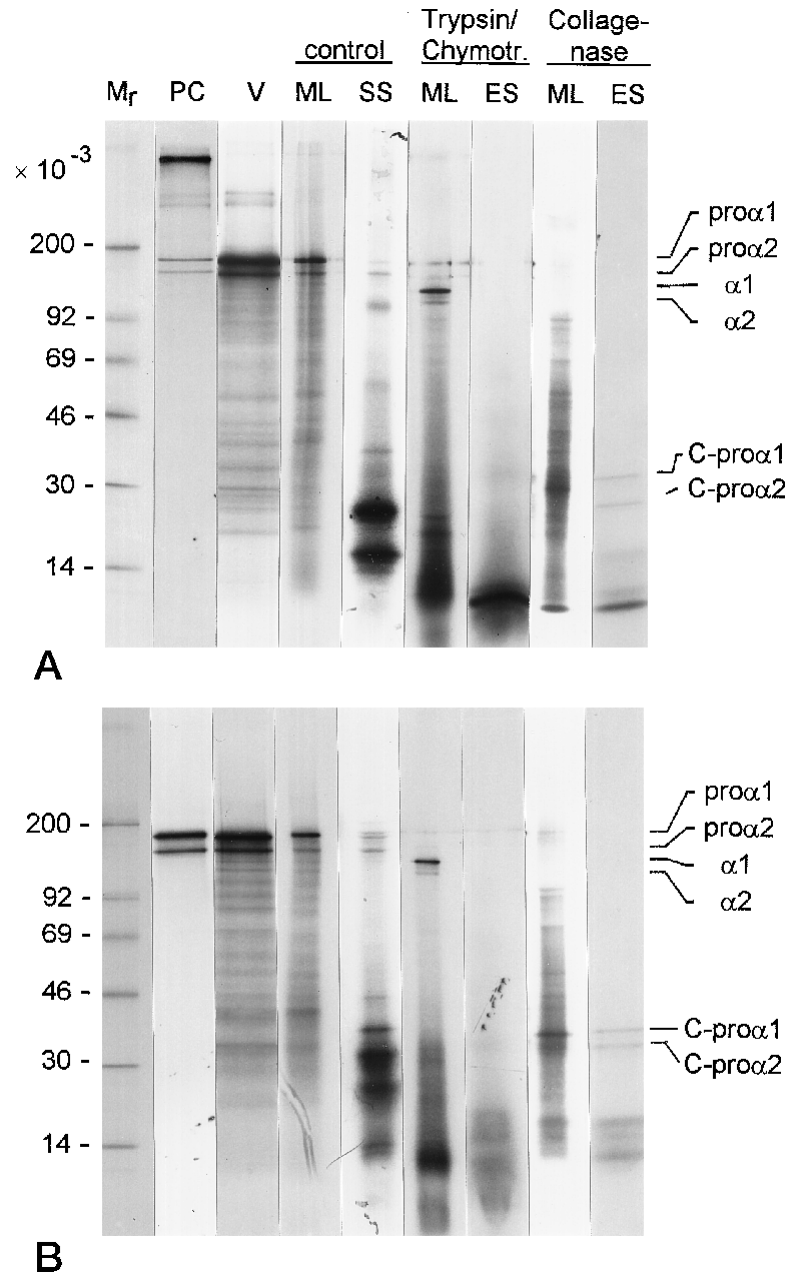

FIG. 6. Fluorograms of material collected from monolayer experiments. Three sets of experiments are shown where samples were run under nonreducing (A) and reducing conditions (B), respectively. $L$ anes $V$ show a representative sample of vesicles used for the experiments, labeled for 7.5 min and treated with NEM. Lanes ML show the collected monolayers, SS, the spreading subphase, and ES, the enzyme containing subphase, of a control experiment and after digestion with trypsin/chymotrypsin or collagenase, respectively. In the case of lanes SS and ES, films were exposed 4-5 times as long as for lanes $V$ and ML. Running positions of marker proteins $\mathrm{M}_{\mathrm{r}}$ and $\left[{ }^{3} \mathrm{H}\right.$ ]Pro-labeled procollagen type I (PC) are included in the left lanes.

been formed in most of the material. Many less sharp bands appear below these which in part might correspond to not fully synthesized procol lagen chains. The monol ayer material exhibits essentially the same pattern, but some lower molecular weight proteins are missing, indicating that these proteins dissolve into the subphase. The spreading subphase contains a low amount of protein including full length procollagen chains. When the total amount of labeled protein from the monolayer and subphase material is compared with the amount of vesicles used for spreading by scintillation counting, the recovery rates turn out to be in the range of $20-40 \%$. Most of the losses probably are due to protein adsorption to the surfaces of instruments involved in the experiments. Despite these limitations, our fluorograms show that a substantial amount of procollagen chains adheres to the transported monolayer.

Protease Stability of Membrane-attached Procollagen-To investigate the folding state of the monolayer-associated collagen chains, we incubated the film on a subphase containing a 9:1 mixture of chymotrypsin/trypsin. Triple helical-folded collagen has been shown to be resistant to cleavage by this enzyme mixture, whereas in its unfolded state it is readily digested 


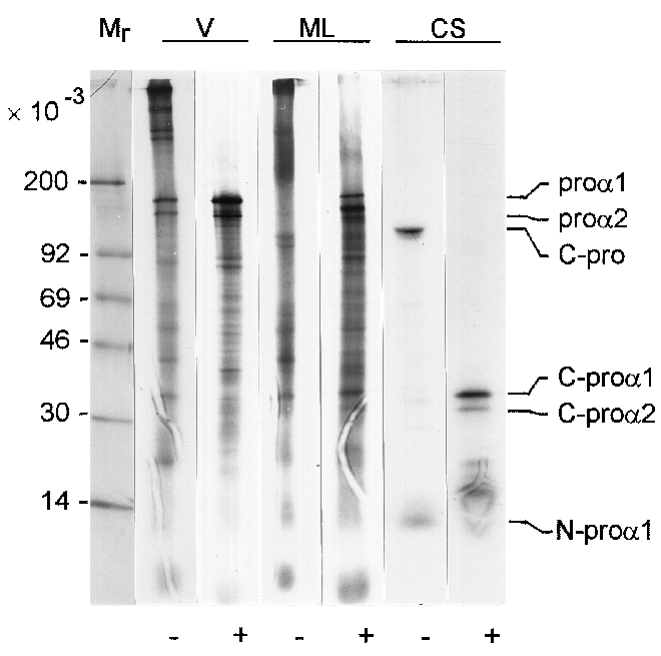

FIG. 7. Fluorograms of collagenase digestion experiments with vesicles not treated with NEM. Lanes show vesicles (V) derived from fibroblasts labeled overnight and which were not treated with NEM, monolayer material after collagenase digestion (ML), and the collagenase-containing subphase (CS) running under nonreducing $(-)$ and reducing $(+)$ conditions, respectively. Running positions of marker proteins are shown in lane $M_{r}$ on the left.

(45). During the incubation on the protease containing subphase, the monolayer area slightly increases due to the high protein concentration (Fig. 4b). Fluorograms of the digested monolayer show an increased electrophoretic mobility and the most distinct bands run in the position of mature collagen type I (Fig. 6). Analogous experiments performed on a $0.1 \mathrm{~m}$ acetic acid subphase using pepsin as protease show essentially the same results (data not shown). This indicates that the procollagen chains attached to the monolayer are fully folded. However, this might not reflect the folding state within the rough $E R$ as triple helix formation could have occurred during vesicle preparation especially upon lowering the temperature. A large amount of labeled material runs in positions of lower molecular mass and is found both attached to the monolayer as well as in the enzyme-containing subphase.

To confirm that the gel bands indeed correspond to collagen, we incubated the monolayer on a subphase containing collagenase (Fig. 4c). Fluorograms of the digested monolayer show a continuum of labeled material running below the position of collagen and procollagen chains, whereas the collagenase-containing subphase shows distinct protein bands in the $M_{r} 30,000$ region (Fig. 6). We performed similar experiments with vesicles which were not treated with NEM. In this case the procollagen chains appear more resistant to collagenase attack (Fig. 7). When run under nonreducing conditions, the bands corresponding to monomeric pro $\alpha$-chains in the digested monolayer nearly vanish, whereas upon reduction clear bands become visible. This indicates that the more stable material corresponds to disulfidelinked pro $\alpha$-chains. Without reduction, the digested monolayer material shows a new band running in a position of $M_{r} \sim 120,000$ which is neither present in the vesicles nor in the monolayer run under reducing conditions. The enzyme-containing subphase reveals a prominent band in the same region which splits into subunits of $M_{r} 33,000$ and 35,000 upon reduction. This behavior corresponds to that of the $\mathrm{COOH}$-terminal propeptide of procollagen type I (46). Furthermore, the subphase includes a substantial amount of a $\mathrm{M}_{r}$ $\sim 11,000$ polypeptide both under reducing and nonreducing conditions which likely represents the amino-terminal propeptide of the pro $\alpha 1$-chain. The pro $\alpha 2$ amino propeptide might either not be visible as it does not contain cysteine and methionine, or it has run out of the gel due to its low size (47). These

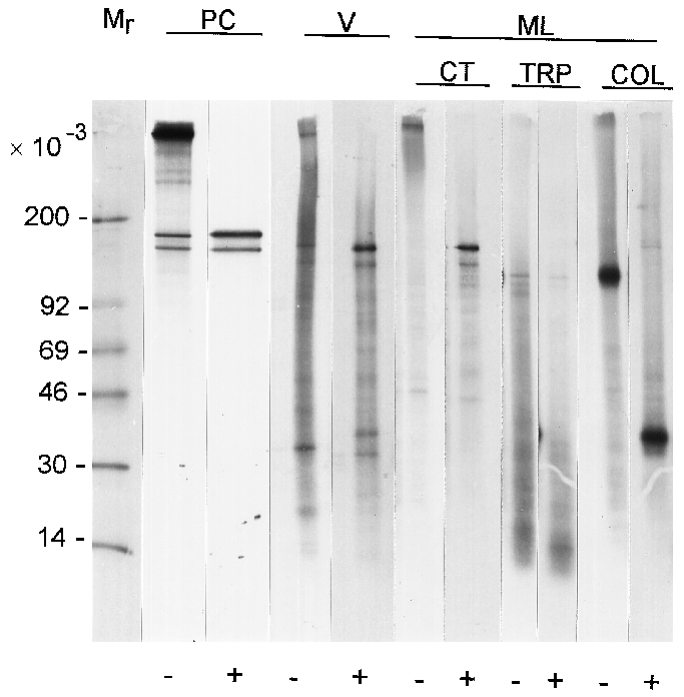

FIG. 8. Fluorograms of protease digestion experiments with vesicles derived from cells treated with $\alpha, \alpha^{\prime}$-dipyridyl and not treated with NEM. Lanes show vesicles (V) derived from fibroblasts treated overnight with $\alpha, \alpha^{\prime}$-dipyridyl and subsequently labeled for 15 min with $\left[{ }^{35} \mathrm{~S}\right] \mathrm{Met},\left[{ }^{35} \mathrm{~S}\right] \mathrm{Cys}$, and $\left[{ }^{14} \mathrm{C}\right]$ Pro. NEM treatment was omitted. Lanes ML show monolayer material recovered as control (CT) and after incubation on chymotrypsin/trypsin (TRP)- and collagenase (COL)-containing subphases running under nonreducing (-) and reducing $(+)$ conditions, respectively. Running positions of marker proteins $\mathrm{M}_{\mathrm{r}}$ and $\left[{ }^{3} \mathrm{H}\right]$ Pro-labeled procollagen type I (PC) are included in the left lanes.

data suggest that the amino propeptide does not interact with the membrane, whereas the carboxyl propeptide interacts weakly with the monolayer.

To test whether triple helix formation is inhibited in our monolayer assay system when hydroxylation and subsequent glycosylation is prevented, we treated cells with $\alpha, \alpha^{\prime}$-dipyridyl. This iron-chelating reagent inhibits prolyl and lysyl hydroxylases $(48,49)$. In this case, no secretion of procollagen is observed due to conformational changes in the procollagen molecule and/or direct inhibition of membrane traffic (50-52). Electrophoretic mobilities of $\alpha 1$ - and $\alpha 2$-procollagen chains were slightly increased compared with those without treatment (Fig. 8), indicating the inhibition of hydroxylation and glycosylation (13). In control monolayer experiments, fluorograms show no significant deviation from those obtained without $\alpha, \alpha^{\prime}$ dipyridyl treatment. Without NEM treatment, the majority of molecules runs in the position of trimeric molecules under nonreducing conditions. Incubation on a chymotrypsin/trypsin containing subphase results in a complete digestion of the molecule indicating that no triple helix has formed (Fig. 8). After collagenase treatment, protein bands with apparent molecular masses of 120,000 and 33,000/35,000 appear under nonreducing and reducing conditions, respectively, which are indicative for the carboxyl-terminal propeptide.

\section{DISCUSSION}

Our data derived from the spreading of microsomes as monolayers present evidence for the hypothesis expressed by Doege and Fessler (15) that folding of the procollagen triple helix occurs when the nascent polypeptide chains are still associated with the membrane. They suggested that as a two-dimensional scaffold the membrane might facilitate sel ective interactions by the telopeptides as a nucleation site for the initiation of folding. For membrane-bound enzymes it has been proposed that turnover rates might increase when their substrate diffusion would be guided by the membrane surface (53). It has been estimated that even at a ratio of three- to two-dimensional diffusion 
coefficients of the order of 100, a transition in kinetics would occur when the reaction compartment and target size are in the micromolar and nanomolar range, respectively (20). The current data on the translational diffusional motion of membrane proteins indeed support the assumed ratio of diffusion coefficient but a critical evaluation reveals that this per se does not speed up reaction rates in the biological systems investigated (54). It is more likely that the increase in local concentration during biosynthesis, when the procollagen I $\alpha$-chains are coordinately synthesized by ER membrane-bound polyribosomes $(21,55)$, increases the probability that appropriate polypeptide chains meet each other. Due to the extended length of the procollagen chains, however, the membrane association might be favorable for correct chain registration.

Most of our experiments have been performed with material which was treated with NEM after labeling. Without this modification, most of our vesicle-enclosed procollagen $\alpha$-chains were disulfide-linked (Figs. 7 and 8). As the chymotrypsin/ trypsin digestion experiments show, the triple hel ix has formed even when for most species interchain disulfide bond formation has been inhibited (Fig. 6A). Initiation of this folding might in part be due to the lowering of temperature and extended time during vesicle preparation. Thus, it might not reflect the conformation after a 7.5-min labeling of the procollagen chains. Nonetheless, it appears that chain selection, registration, and correct folding can occur while the pro $\alpha$-chains are still associated with the ER membrane. This suggests a different mechanisms from that observed in in vitro folding studies of the fibril-forming collagens I to III in solution. When processed collagen I is thermally denatured and refolded upon cooling, the yield of native molecules is extremely small (for reviews, see Refs. 56 and 57). For mature collagen type III, a nearly complete and correct refolding could be observed (10, 12). This implied that the interchain disulfide bonds between all three chains near the carboxyl terminus are responsible for chain registration. A similar role was assumed for the disulfide bridges present in the carboxyl-terminal propeptides of procollagen I and II. Our data do not contradict the in vivo observation that, in the case of collagen I, a heterotrimeric complex of pro $\alpha$-chains becomes first stabilized by interchain disulfide bonds before folding of a triple helix occurs $(7,8,58,59)$. The observation that a protease-resistant complex of pro $\alpha$-chains is formed even when the formation of an interchain disulfide linkage is prevented by NEM treatment (Fig. 6A), however, suggests that triple helix formation is an independent process. This view is supported by our experiments in which hydroxylation and subsequent glycosylation is inhibited by $\alpha, \alpha^{\prime}$-dipyridyl. In this case the carboxyl-terminal interchain disulfide bonds in the propeptides can form without triple helix formation (Fig. 8). Interestingly, in the case of collagen types IV and XII, which do not form fibrils, it has been recently proposed that lateral aggregation via the triple helical domains and/or hydroxylation is essential for proper interchain disulfide linkage between the weakly interacting carboxyl-terminal domains NC1 $(60,61)$ (see, however, Davis et al. (17)).

We presently do not know how the procollagen chains are associated with the membrane. In contrast to other in vitro studies, our assay system ensures that the proteins present during the biosynthesis of procollagen type I are also included in all phases of the experiments. Thus we cannot exclude that other proteins are involved in a linkage of the procollagen chains to the membrane. For technical reasons, we have chosen a labeling time of $7.5 \mathrm{~min}$, which is about the time needed for the biosynthesis of complete pro $\alpha$-chains $(35,36)$. This assures that we obtain a maximum labeling rate but still see mainly procollagen chains which synthesis has been just completed or is still in progress. Even with the use of [ $\left.{ }^{35} \mathrm{~S}\right] \mathrm{Met},\left[{ }^{35} \mathrm{~S}\right] \mathrm{Cys}$, and $\left[{ }^{14} \mathrm{C}\right]$ Pro as label, we have worked near the practical limits of detection by fluorography as our film exposure times were up to 7 weeks. Due to our nonspecific labeling procedure we could also observe the presence of several proteins most probably unrelated to collagen in our vesicle preparations (Figs. 6 and 7). Among others, we consistently found distinct protein bands corresponding to a $M_{r}$ of $\sim 47,000$ and 35,000. Preliminary analysis of the vesicle material by Western blotting indicates that the 47-kDa band might correspond to HSP47/colligin. ${ }^{2}$ HSP47 has been attributed to assist in the correct folding and packaging of procollagen during biosynthesis and transport, although its biological function remains unclear $(62,63)$.

Another possibility for the association of procollagen chains with the ER membrane might be a specific interaction with lipids. Several types of lipids, including phosphatides, cholesterol, plasmalogens, and gangl iosides, have been found even in highly purified collagen preparations $(64,65)$, and in vitro studies suggested a specific binding of collagen type I to phosphatidylcholine (66). A more recent analysis using density centrifugation of various lipid vesicles incubated with procollagen type I, however, could not find any specific lipid binding except for high concentrations of sphingomyelin, and only when this is in the gel-crystalline phase (40). This interaction could be attributed to the carboxyl-terminal propeptide. When we chose POPC as a lipid for the preformed monolayer to prevent surface denaturation, we tested its interaction capability with collagen type I purified from calf skin. In monolayer experiments performed at constant surface area in analogy to the experiments shown in Fig. 3, we could observe a limiting surface pressure of only $22 \mathrm{mN} / \mathrm{m}$ (data not shown). Based on these measurements we expect that our initial POPC monolayer will not interfere as an artificial substrate for procollagen association.

In summary, our data present evidence that chain recognition, registration, and triple helix folding of the procollagen type I molecule occur when the polypeptide chains are still in close association with the rough ER membrane, and these processes are independent of the formation of interchain disulfide bonds. However, it remains an open question whether the membrane interaction is mediated by other molecules and whether additional proteins participate in the folding process.

Acknowledgment-We gratefully acknowledge Douglas R. Keene for his continuous support and critical advice with electron microscopy.

\section{REFERENCES}

1. Kadler, K. (1995) Protein Profile 2, 491- 619

2. Ramachandran, G. N., and Reddi, A. H. (eds) (1976) Biochemistry of Collagen, Plenum Press, New York

3. Olson, B. R. (1991) in Cell Biology of the Extracellular Matrix (E. D. Hay, ed) pp. 177-220, Plenum Press, New York

4. Brownell, A. G., and Veis, A. (1976) J . Biol. Chem. 251, 7137-7143

5. Bornstein, P. (1967) Biochemistry 6, 3082-3093

6. Bornstein, P. (1967) J . Biol. Chem. 242, 2572-2574

7. Byers, P. H., Click, E. M., Harper, E., and Bornstein, P. (1975) Proc. Natl. Acad. Sci. U. S. A. 72, 3009-3013

8. Lukens, L. N. (1976) J . Biol. Chem. 251, 3530-3538

9. Bächinger. H. P., Bruckner, P., Timpl, R., and Engel, J . (1978) Eur. J . Biochem. 90, 605- 613

10. Bächinger, H. P., Bruckner, P., Timpl, R., Prockop, D. J ., and Engel, J . (1980) Eur. J . Biochem. 106, 619-632

11. Engel, J., and Prockop, D. J . (1991) Annu. Rev. Biophys. Biophys. Chem. 20, 137-152

12. Bächinger, H. P., Fessler, L. I., Timpl, R., and Fessler, J . H. (1981) J . Biol. Chem. 256, 13193-13199

13. Fessler, L. I., and Fessler, J . H. (1974) J . Biol. Chem. 249, 7637-7646 14. Fessler, L. I., Timpl, R., and Fessler, J. H. (1981) J. Biol. Chem. 256, 2531-2537

15. Doege, K. J ., and Fessler, J . H. (1986) J . Biol. Chem. 261, 8924- 8935

16. Bächinger, H. P. (1987) J . Biol. Chem. 262, 17144-17148

17. Davis, J. M., Boswell, B. A., and Bächinger. H. P. (1989) J . Biol. Chem. 264, 8956- 8962

18. Steinmann, B., Bruckner, P., and Superti-Furga, A. (1991) J . Biol. Chem. 266,

\footnotetext{
${ }^{2}$ B. Boswell, unpublished results.
} 
$1299-1303$

19. Bächinger, H. P., Morris, N. P., and Davis, J . M. (1993) Am. J . Med. Genet. 45, 152-162

20. Adam, G., and Delbrück, M. (1968) in Structural Chemistry and Molecular Biology (Rich, A., and Davidson, N., eds) pp. 198-215, Freeman, San Francisco

21. Veis, A., Leibovich, S. J ., Evans, J ., and Kirk, T. Z. (1985) Proc. Natl. Acad. Sci. U. S. A. 82, 3693-3697

22. Peterkofsky, B., and Diegelmann, R. (1971) Biochemistry 10, 988-994

23. Bond, M. D., and Van Wart, H. E. (1984) Biochemistry 23, 3085-3091

24. Dehm, P., and Prockop, D. J . (1971) Biochim. Biophys. Acta 240, 358-369

25. Kao, W. W.-Y., Berg, R. A., and Prockop, D. J. (1977) J . Biol. Chem. 252, 8391- 8397

26. Stafford, F. J ., and Bonifacino, J. S. (1991) J . Cell Biol. 115, 1225-1236

27. Storrie, B., and Madden, E. A. (1990) Methods Enzymol. 182, 203-225

28. Green, M., and Alper, B. J . (1979) Arch. Biochem. Biophys. 195, 368-377

29. Fromherz, P. (1975) Rev. Sci. Instrum. 46, 1380-1385

30. Gaines, G. L., J r. (1966) I nsoluble Monolayers at Liquid-Gas I nterfaces, Interscience Publishers, New York

31. Trurnit, H. J . (1960) J . Colloid Sci. 15, 1-13

32. Verger, R., and Pattus, F. (1976) Chem. Phys. Lipids 16, 285-291

33. Laemmli, U. K. (1970) Nature 227, 680-685

34. Sakai, L. Y., and Keene, D. R. (1994) Methods Enzymol. 245, 29-52

35. Bruckner, P., Eikenberry, E. F., and Prockop, D. J . (1981) Eur. J . Biochem 118, 607- 613

36. Fessler, L. I., Morris, N. P., and Fessler, J . H. (1975) Proc. Natl. Acad. Sci. U. S. A. 72, 4905- 4909

37. White, D. A. (1973) in Form and Function of Phospholipids (Ansell, G. B. Hawthorne, J. N., and Dawson, R. M. C., eds) pp. 441-482, Elsevier Scientific, Amsterdam

38. Hofer, A. M., Schlue, W.-R., Curci, S., and Machem, T. E. (1995) FASEB J . 9, 788-798

39. Schürholz, T., and Schindler, H. (1991) Eur. Biophys. J . 20, 71-78

40. Choglay, A. A., Purdom, I. F., and Hulmes, D. J . S. (1993) J . Biol. Chem. 268, 6107- 6114

41. Smaby, J. M., and Brockman, H. L. (1985) Biophys. J . 48, 701-708

42. Schindler, H. (1979) Biochim. Biophys. Acta 555, 316-336

43. Demel, R. G., Geurts van Kessel, W. S. M., Zwaal, R. F. A., Roel ofsen, B., and
Van Deenen, L. L. M. (1975) Biochim. Biophys. Acta 406, 97-107

44. Chapman, D., Gomez-Fernandez, J. C., and Goni, F. M. (1982) Trends Biochem. Sci. 7, 67-70

45. Bruckner, P., and Prockop, D. J . (1981) Anal. Biochem. 110, 360-368

46. Pedersen, B. J ., and Bonde, M. (1994) Clin. Chem. 40, 811-816

47. Morris, N. P., Fessler, L. I., and Fessler, J. H. (1979) J . Biol. Chem. 254, 11024-11032

48. J uva, K., Prockop, D. J ., Cooper, G. W., and Lash, J . W. (1966) Science 152, 92-94

49. Margolis, R. L., and Lukens, L. N. (1971) Arch. Biochem. Biophys. 147, 612- 618

50. J imenez, S., Harsch, M., and Rosenbloom, J . (1973) Biochem. Biophys. Res. Commun. 52, 106-114

51. Berg, R. A., and Prockop, D. J . (1973) Biochem. Biophys. Res. Commun. 52 115- 120

52. J imenez, S. A., and Yankowski, R. (1978) J . Biol. Chem. 253, 1420-1426

53. Trurnit, H. J . (1945) Fortschr. Chem. Org. Naturst. 4, 347- 476

54. McCloskey, M. A and Poo, M.-M. (1986) J. Cell Biol, 102, 88-96

55. Veis, A., and Kirk, T. Z. (1989) J . Biol. Chem. 264, 3884-3889

56. Engel, J . (1987) in Advances in Meat Research (Pearson, A. M., Dutson, T. R., and Bailey, A. J., eds) Vol. 4, pp. 145-158, Van Nostrand Reinhold, New York

57. Traub, W., and Piez, K. A. (1971) Adv. Protein Chem. 25, 243-352

58. Morris, N. P., Fessler, L. I., Weinstock, A., and Fessler, J. H. (1975) J . Biol Chem. 250, 5719-5726

59. Schofield, J. D., Uitto, J., and Prockop, D. J. (1974) Biochemistry 13, 1801-1806

60. Mazzorana, M., Giry-Lozinguez, C., and van der Rest, M. (1994) Matrix Biol 14, 583-588

61. Ries, A., Engel, J., Lustig, A., and Kühn, K. (1995) J . Biol. Chem. 270 23790-23794

62. Hu, G., Gura, T., Sabsay, B., Sauk, J ., Dixit, S. N., and Veis, A. (1995) J . Cell. Biochem. 59, 350-367

63. Nagata, K. (1996) Trends Biochem. Sci. 21, 23-26

64. Rabinovitz, J. L., and Shapiro, I. M. (1972) Arch. Oral Biol. 17, 547-553

65. Özgünes, N., and Artvinli, S. (1988) Biochem. Med. Metab. Biol. 40, 269-275

66. Martinez del Pozo, A., Oñaderra, M., Laynez, J ., and Gavilanes, J . G. (1988) Collagen Relat. Res. 8, 133-144 